\title{
Modified Compact Combline Filter Using Planar Parallel Coupled Structure with Extended Rejection Bandwidth
}

\author{
In-Ho Kang* • Kai Wang** · Shang MIng Li*** \\ *,**,*** Dept. of Radio Engineering Korea Maritime University
}

\begin{abstract}
Modified compact combline bandpass filters are proposed based on the miniaturized quarter-wave transmission line which is composed of the parallel coupled line and lumped capacitors. The electrical length of the parallel coupled line in a resonator, which determines the size of combline bandpass filters, is just $5^{\circ}$ or $7^{\circ}$, resulting in a compact circuit area. The designed combline bandpass filter also has a wide upper stopband by suppressing the spurious passbands, not moving. Measured results of two fabricated filters centered at $400 \mathrm{MHz}$ show good agreement with the theoretical predications.
\end{abstract}

Key words : Miniaturization; combline filter; parallel coupled line

\section{Introduction}

Microwave bandpass filters with compact size and low cost are intensely required in modern mobile wireless communication systems because miniaturization is in great demand for most mobile platforms. The parallel coupled line filter proposed by Cohn (1958)has been widely used in microwave systems over the last decades due to its planar structure, simple synthesis procedures and fabrication facility. Nevertheless, these conventional parallel coupled line filters are too large to apply to mobile communication systems. To reduce the size, folded hairpin resonator filters(Hong, 1998 ; Sagawa, 1989 ; Djaiz, 2006)and stepped-impedance filters were developed (Makimoto, 1980 ; Kuo, 2003 ; Wang, 2004 ; Kong, 2004), and slow wave open-loop resonator filters have been investigated(Hong, 1997 ; Hong, 1996 ; Piston, 2006). Using the above proposed methods, compact bandpass filter can be achieved. However, they still take up quite a large circuit area, especially at lower microwave frequency band.

Another disadvantage of the traditional parallel coupled line filters is that the first spurious passband of this type of filter appears at twice of the basic passband frequency $\left(2 f_{0}\right)$, which may seriously degrade the attenuation level in the stopband and passband response symmetry. This is because the phase velocity of the even mode is always slower than that of the odd mode due to the inhomogeneous medium of microstrip structure. The unequal modal phase velocities at $2 f_{0}$ cause the first spurious passband and this could limit the applicability of the filter. Many papers have been proposed to overcome this problem. To compensate slow even mode velocity, the parallel coupled line filters with over coupled line stages is proposed to extend the electrical length of odd mode (Kuo, 2003). A proper height of substrate suspension can be used to equalize the even- and odd-mode phase velocities in the parallel coupled line filters(Kuo, 2004). In wiggly-line filter structure, wave impedance using the continuous perturbation of the width of the coupled line can be modulated so that the harmonic passband is rejected(Lopetegi, 2001). Meandered parallel coupled line filters also effectively suppress the spurious passband(Wang, 2005). However, the first spurious passband of these published filters is just moved to the higher frequency, and not removed. The upper spurious passbands still exist in these filters.

Combline filters using low temperature $\mathrm{co}^{-}$-fired ceramic (LTCC) or the ceramic material with the feature of three dimensional structures can be used as reduced size bandpass filters(Tang, 2003 ; Tang, 2006 ; Yao, 1996). Conventionally, the electrical length of combline filters has been recommended as $45^{\circ}$ or less for efficient coupling (Matthaei, 1980). Moreover, it is not straightforward to calculate the self- and mutual-capacitances for the combline filter with lumped components, and an accurate analysis of the combline structure is a required procedure.

The modified comb-line filter with the electrical length of

\footnotetext{
* ihkang@hhu.ac.kr, 051)410-4422

** lovekmu@hotmail.com, 051)410-4919

***yanminv@live.cn, 051)410-4919
} 
$10^{\circ}$ was published (Wang, 2009). This paper was focused on the harmonics suppression for the power amplifier efficiency improvement, not highly miniaturizing the filter.

For the compact size design, the bandpass filter with lumped elements also can be a solution. But this lumped-element approach is somewhat empirical and has been confined to frequencies up to a few $\mathrm{GHz}$ due to the low quality factor and low resonant frequencies (Ju, 1984).

In this paper, a new method will be introduced to miniaturize the size of bandpass filters to as small as a few degrees. There is no spurious in this structure. For the simple fabrication, modified combline filter with the planar microstrip structure will be used.

This paper is organized as follows: In section 2, the limitations of the conventional stepped-impedance resonator will be introduced. Then miniaturized quarter-wave transmission line is introduced in section 3, which plays a key role in building the proposed bandpass filter. Following section 3 is the design method of the bandpass filter in Section 4. To get better performance some tunings are needed before fabrication and this is discussed in Section 5 . Also presented in section 5 is the measured results of two fabricated bandpass filters centered at $400 \mathrm{MHz}$. Conclusions are in section 6 .

\section{Limitations of Stepped-Impedance Resonator Filters}

Stepped-impedance resonator bandpass filter has been studied for a compact size. Fig. 1 gives the basic structure of half-wave stepped-impedance resonator filters. It consists of two kinds of transmission lines with the characteristic impedances being $Z_{1}$ and $Z_{2}$. The high-impedance lines act as series inductors, while the low- impedance lines act as shunt capacitors. The input admittance $Y_{i}$ seen from an open-end is(Makimoto, 1980):

$Y_{i}=j Y_{2}$

$$
\frac{2\left(R_{Z} \tan \theta_{1}+\tan \theta_{2}\right)\left(R_{Z}-\tan \theta_{1} \tan \theta_{2}\right)}{R_{Z}\left(1-\tan ^{2} \theta_{1}\right)\left(1-\tan ^{2} \theta_{2}\right)-2\left(1+R_{Z}^{2}\right) \tan \theta_{1} \tan \theta_{2}}
$$

where the impedance ratio $R_{Z}$ is defined by

$$
R_{Z}=\frac{Z_{2}}{Z_{1}}
$$

Resonance condition is obtained by taking $Y_{i}=0$, thus giving:

$$
R_{Z}=\tan \theta_{1} \tan \theta_{2}
$$

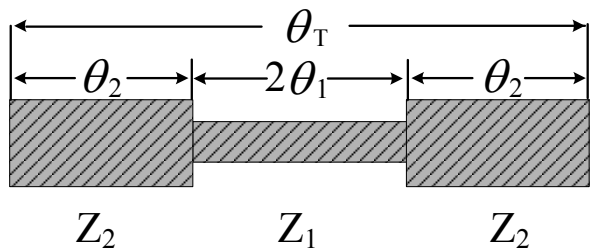

Fig. 1 Basic structure of a stripline $\lambda_{g} / 2$ type stepped-impedance resonator.

The relationship between the total electrical length $\theta_{T}$ of the resonator and $\theta_{1}, \theta_{2}$ is given by

$$
\begin{aligned}
\theta_{\mathrm{T}} & =2\left(\theta_{1}+\theta_{2}\right) \\
& =2\left(\theta_{1}+\tan ^{-1}\left(\frac{R_{Z}}{\tan \theta_{1}}\right)\right), R_{Z} \neq 1
\end{aligned}
$$

From (4), it can be shown that to obtain the smallest filter size, we should choose a very small value of $R_{Z}$. This can be done by decreasing $Z_{2}$ and increasing $Z_{1}$. However, if $Z_{1}$ becomes very large, it is very difficult to fabricate the circuit. Therefore, stepped-impedance resonator filters have limitations in size-reduction.

\section{Miniaturization of Quarter-Wave Transmission Line}

Before starting our filter design theory, the size-reduction method of a $N / 4$ transmission line will be briefly introduced here(Kang, 2003). As is well known, the line can be replaced with a lumped circuit, as given in Fig. 2, and the value of $C_{1}$ is given by

$$
C_{1}=\frac{1}{\omega Z_{0}}
$$

where $Z_{0}$ is the characteristic impedance of the quarter-wave transmission line and $\omega$ the angular frequency.

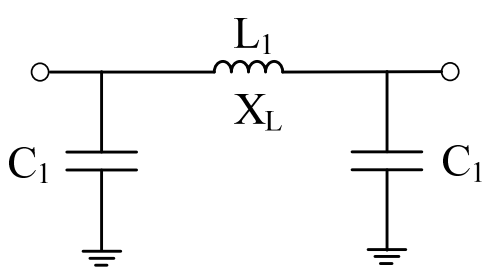

Fig. 2 The equivalent lumped circuit of the quarter-wave transmission line. 
Now to replace the lumped inductor, we add two artificial LC resonators consisting of $\mathrm{C}_{0}$ and $\mathrm{L}_{0}$ at each side of the lumped inductor, and then we can get Fig. 3. The dotted network in Fig. 3 is equal to the circuits in Fig. 4 (a) and (b) when (6) and (7) are satisfied.

$$
\begin{aligned}
& X_{0}=Z_{0 e} \tan \theta \\
& X_{L}=\frac{2 Z_{0 e} Z_{0 o}}{Z_{0 e}-Z_{0 o}} \tan \theta
\end{aligned}
$$

where $Z_{0 e}, Z_{0 o}=$ even and odd mode impedances of the coupled line, respectively, and $\theta$ is the electrical length of parallel short-ended coupled line.

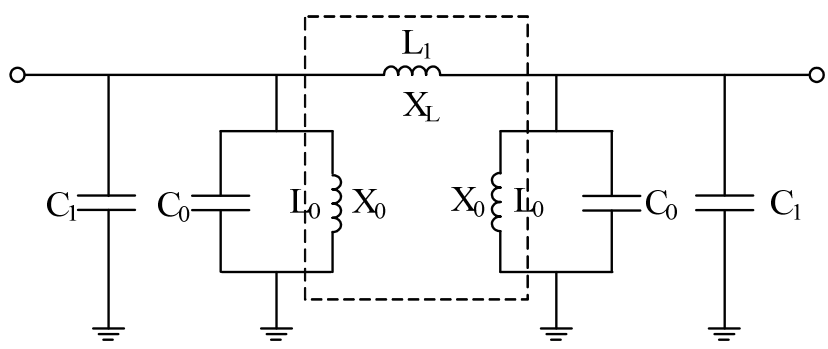

Fig. 3 The equivalent lumped circuit of the quarter-wave transmission line with two resonators added.

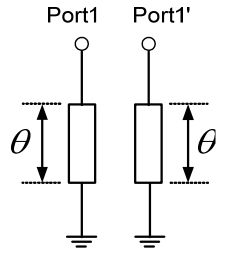

(a)

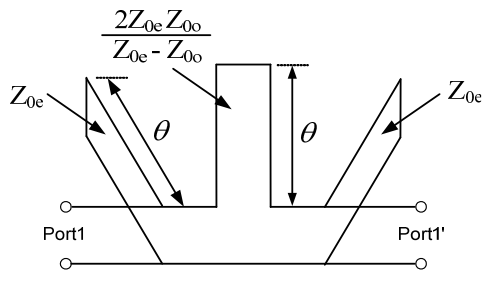

(b)

Fig. 4 Short-ended coupled line (a) and its equivalent network (b).

With (6) and (7), the three inductors in Fig. 3 can be replaced with the short-ended coupled line, resulting in the circuit in Fig. 5. And the value of $\mathrm{C}_{0}$ can be deduced from (6) as:

$$
C_{0}=\frac{1}{\omega Z_{0 e} \tan \theta}
$$

$$
\text { because } X_{0}=\omega L_{0}=\frac{1}{\omega C_{0}}
$$

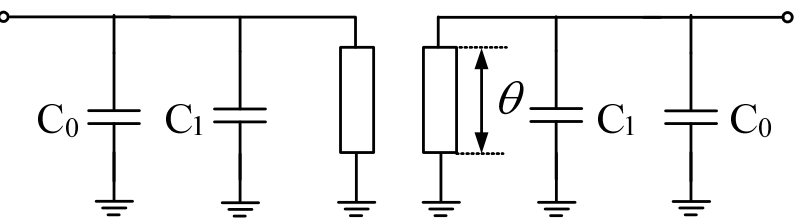

Fig. 5 The miniaturized quarter-wave transmission line with short-ended coupled line and lumped capacitors.

In the following simulation and fabrication, one capacitor $\mathrm{C}$ will be used as the sum of $\mathrm{C}_{0}$ and $\mathrm{C}_{1}$, and the final simplified circuit of the quarter-wave transmission line is expressed in Fig. 6. Equation (10) will be used in this paper to calculate the value of $\mathrm{C}$ :

$$
C=\frac{1}{\omega Z_{0}}+\frac{1}{\omega Z_{0 e} \tan \theta}
$$

Fig. 6 Final miniaturized quarter-wave transmission line.

If defining $Z^{\prime}=\frac{2 Z_{0 e} Z_{0 o}}{Z_{0 e}-Z_{0 o}}$ as the characteristic impedance of the short-ended coupled line, then with (7) we get:

$$
Z^{\prime}=\frac{Z_{0}}{\tan \theta}=\frac{2 Z_{0 e} Z_{0 o}}{Z_{0 e}-Z_{0 o}}
$$

When the electrical length $\theta$ is very small for compact size, $Z^{\prime}$ becomes very large, e.g. $Z^{\prime}$ is $407 \Omega$ when $\theta=7^{\circ}$ and $Z_{0}=50 \Omega$. This large $Z^{\prime}$ can be easily achieved by making $Z_{0 e}$ and $Z_{0 o}$ nearly the same.

\section{Bandpass Filter Design}

The bandpass filter to be designed aims at center frequency $=400 \mathrm{MHz}$ and fractional bandwidth $w=10 \%$. Firstly, the generalized bandpass filter model provided by Matthaei (1980)is employed to design the target filter, as shown in Fig. 7. A bandpass filter with two resonators based on this model is offered in Fig. 8 (a), with the help 
of the following equations $(12) \sim(16)$ :

$$
\begin{aligned}
& J_{01}=\sqrt{\frac{Y_{A} l_{1} w}{g_{0} g_{1} w_{l}{ }^{\prime}}} \\
& \left.J_{j, j+1}\right|_{j=1 \text { to } n-1}=\frac{w}{w_{1}^{\prime}} \sqrt{\frac{l_{j} l_{j+1}}{g_{j} g_{j+1}}} \\
& J_{n, n+1}=\sqrt{\frac{Y_{B} l_{n} w}{g_{n} g_{n+1} w_{l}^{\prime}}} \\
& l_{j}=\omega_{0} C_{j}=\frac{1}{\omega_{0} L_{j}} \quad(j=1,2, \ldots ., n) \\
& w_{1}{ }^{\prime}=1
\end{aligned}
$$

where $J_{01}, J_{j, j+1}$ and $J_{n, j+1}=$ inverter parameters; $l_{j}=$ susceptance slope parameters;

$g_{j}=$ element values in Chebyshev low-pass prototype; $C_{j}, L_{j}=$ lumped capacitors and inductors.

Then the admittance inverter, which is quarter-wave transmission line with normalized characteristic admittance $J_{j, j+1}$ can be miniaturized using the method introduced in last section, as shown in Fig. 8 (a), (b), where $\theta_{j, j+1}$ is the electrical length of each stage in the miniaturized filter and $C_{j, j+1}$ is the corresponding capacitors. As two examples, here we reduced the electrical length $\theta_{j, j+1}$ to 45 and 7 degrees, respectively. However, Fig. 8 (c) shows that the size-reduced bandpass filters have narrower bandwidth than that of the conventional one. According to Fig. 8 (c), the bandwidth of conventional bandpass filter with $-1 \mathrm{~dB}$ insertion loss is $45 \mathrm{MHz}$. The miniaturized one with $\theta_{j, j+1}=45^{\circ}$ is $38 \mathrm{MHz}$ and $\theta_{j, j+1}=7^{\circ}$ is $29 \mathrm{MHz}$. Due to this bandwidth shrinkage the miniaturized filter deviated from the target.

In addition, the adoption of generalized bandpass filter design method results in different values of inverter characteristic admittance $J_{j, j+1}$. Thus, the capacitors $C_{j, j+1}$ also have different values. This is not convenient since the commercialized lumped capacitors can not support the various capacitor values. And because some other artificial resonators were inserted in the procedure of miniaturization, the designed susceptance slope parameters already changed. Therefore the original resonators became unnecessary. For these reasons, we will not adopt the generalized bandpass filter model for designing extremely miniaturized bandpass filter.
Instead, the circuit in Fig. 9 is considered to be the model that can be miniaturized using the size-reduction method introduced in section 3. More stages can be achieved simply by connecting several of the same circuits. To prove this discussion, ADS is used to simulate $1^{-}, 2^{-}$ and 3-stage proposed compact bandpass filters. It is worth noting that the stage number mentioned in this paper is not determined by the resonators as usual, but rather it is the

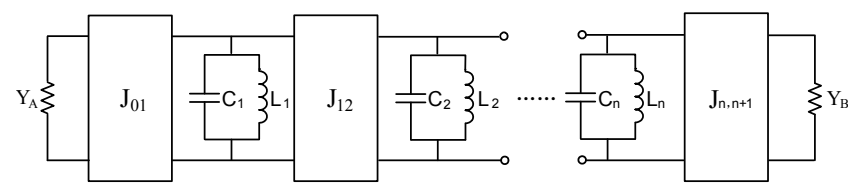

Fig. 7 A generalized bandpass filter circuit using admittance inverters.

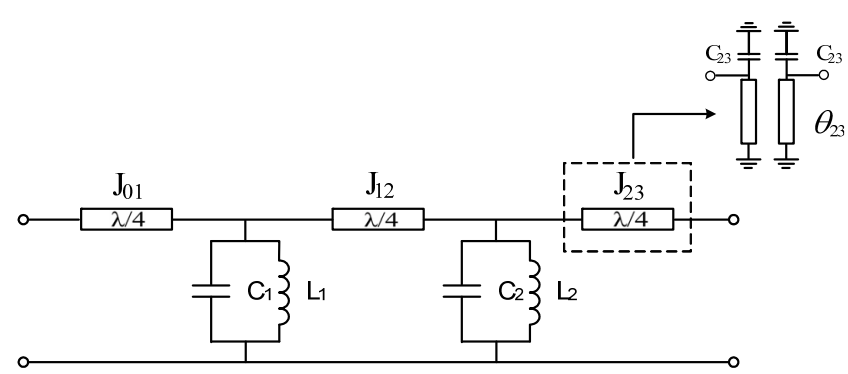

(a)

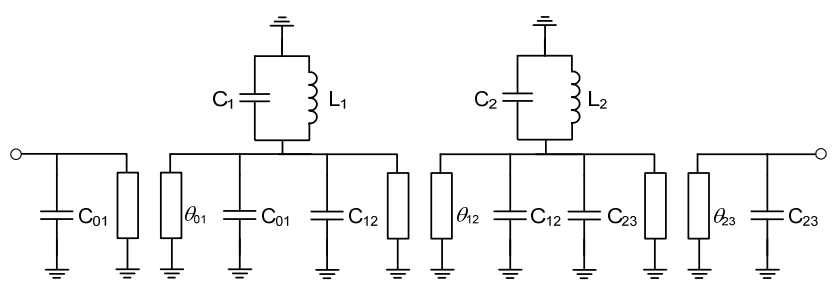

(b)

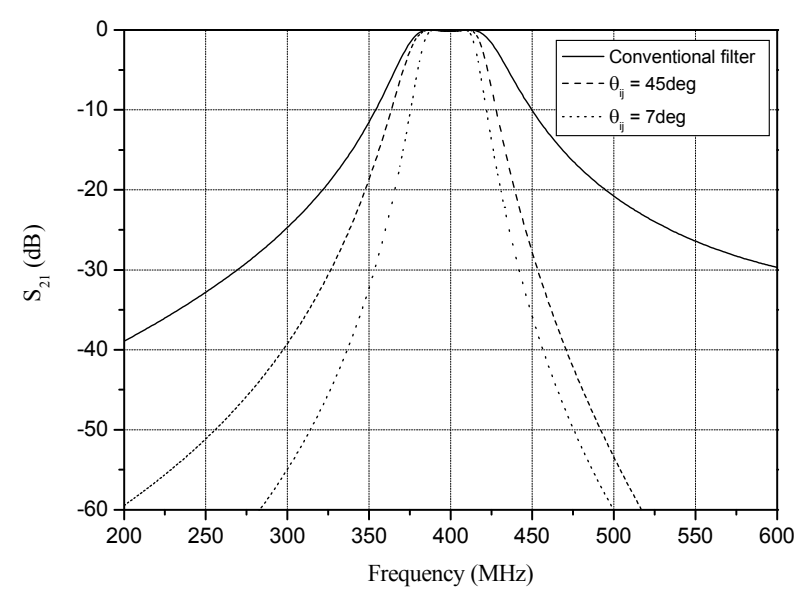

(c)

Fig. 8 Designed filter based on the generalized bandpass filter. (a) Conventional filter, (b) miniaturized filter, (c) simulated results. 


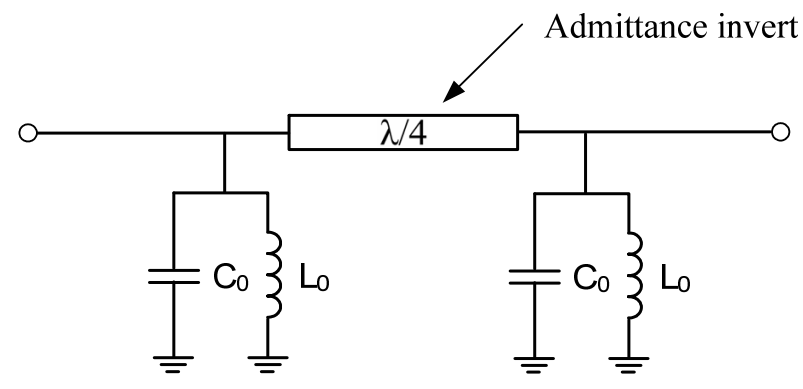

Fig. 9 Model of one-stage bandpass filter used in this paper.
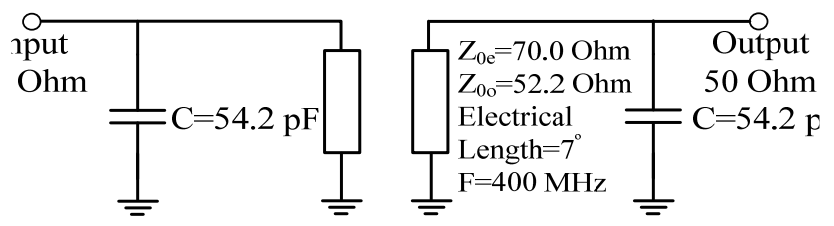

(a)

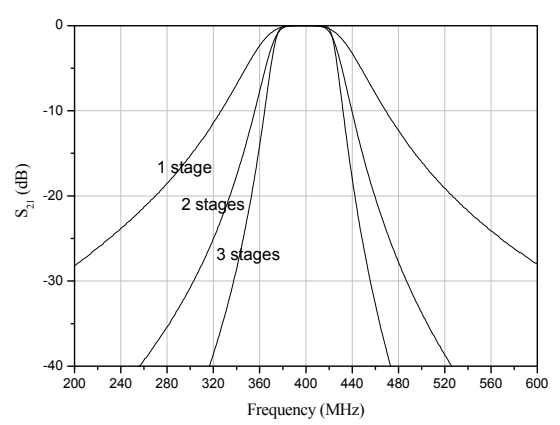

(b)

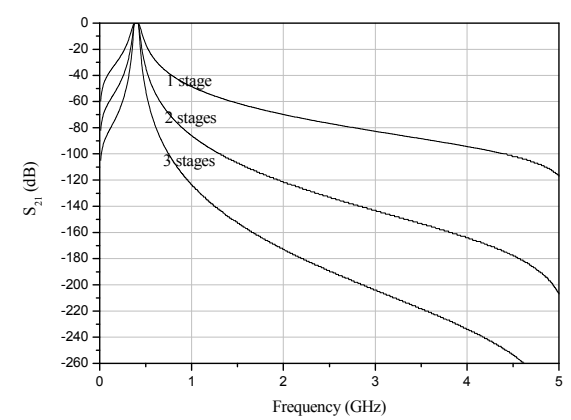

(c)

Fig. 10 (a) ADS model of the miniaturized 1-stage 7-degree filter, (b) simulated results of $1^{-}, 2^{-}$and $3-$ stage 7-degree filters for comparison of slope characteristics, (c) broadband characteristics.

inverter number. In other words, an $\mathrm{n}^{-}$-stage miniaturized filter means it consists of $\mathrm{n}$ pairs of coupled line, and the inverter number of original filter is $\mathrm{n}$. Since the port characteristic impedance is $50 \Omega$, the inverter characteristic impedance should also be $50 \Omega$. The electrical length of the coupled line in miniaturized filter was chosen to be 7 degrees, and by fixing $Z_{0 e}$ as $70 \Omega$, we can get the value of $Z_{0 o}$ as $52.2 \Omega$ using equation (11). The capacitor value was obtained with (10), and is given in Fig. 10 (a). The skirt characteristic improves as more stages are connected in series, shown in Fig. 10 (b), but in fabrication the use of many stages will inevitably result in a large insertion loss.

The broadband characteristics in Fig. 10 (c) show good suppression of spurious passbands. This is because the lumped capacitors are used as resonators while the conventional bandpass filter has the structure that half-wave transmission lines are used as resonators. Therefore, these lumped capacitors suppress the harmonics resulting from distributed circuits effectively.

Bandwidth is an important parameter of bandpass filter. The bandwidth of the proposed filter is closely related to the coupling coefficient $K=\frac{Z_{0 e}-Z_{0 o}}{Z_{0 e}+Z_{0 o}}$ of the short-ended coupled line(Kang, 2003). The larger the coupling coefficient $\mathrm{K}$, the broader the bandwidth of the filter, as illustrated in Fig. 11, where the electrical length of the coupled line is chosen as $7^{\circ}$ and two stages are used. When we miniaturize the quarter-wave transmission line, we can choose a proper coupling coefficient according to the bandwidth of the required bandpass filter. However, to achieve a broad bandwidth, the coupling coefficient $\mathrm{K}$ should be made as large as possible, which means the difference between $Z_{0 e}$ and $Z_{0 o}$ should be large. It will result in a small $Z^{\prime}$ and hence a large electrical length of the coupled line. Therefore, a trade-off is needed between broad bandwidth and small circuit size.

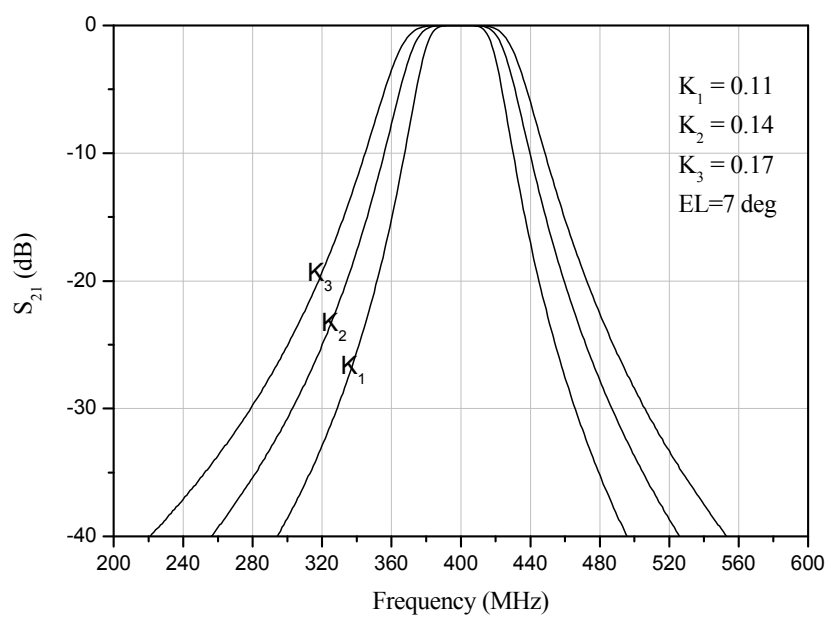

Fig. 11 Relation between the bandwidth and coupling coefficient of the coupled line (electrical length of the coupled line is chosen as $7^{\circ}$ and two stages are used). 
The proposed bandpass filter can be categorized as combline filters. However, it has the following new features:

Firstly, compared with conventional combline filters, an inter-stage transmission line has been used to prevent the unwanted coupling between the two neighboring resonators. And distortions will appear if this inter-stage transmission line is too short or not used. The further discussion is in the next section.

Secondly, there are resonance circuits at both the input and output ports. In a traditional combline filter, the short-ended transmission lines used as input and output are equivalent to shunt inductors, as illustrated in Fig. 12 (Matthaei, 1980). If $\theta$ is small, inductance $\mathrm{L}$ is tremendously small and nearly short. Therefore, the signals can not flow into the filter, which hindered the miniaturization of the traditional combline filters. Actually, the electrical length of the traditional combline filter was generally recommended as $4^{\circ}$ or less in(Matthaei, 1980). On the contrary, in this new design theory $\# 0$ and $\# n+1$ resonators naturally appear because artificial resonators were added for the purpose of miniaturizing quarter-wave transmission line.

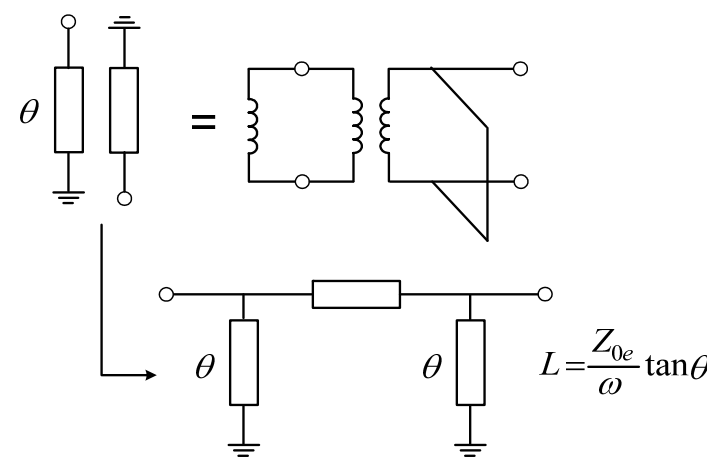

Fig. 12 Equivalent circuit of the iutput and ouput sections in the conventional combline filters.

Thirdly, the size of the parallel coupled line in the modified combline filter can be extremely miniaturized according to $Z^{\prime} \frac{2 Z_{0 e} Z_{0 e}}{Z_{0 e}-Z_{0 e}}$, so long as we choose $Z_{0 e} \approx Z_{0 o}$. No combline filter as small as a few degrees has been published, to the authors' knowledge.

A compact bandpass filter is found in (Kang, 2007), which is miniaturized based on Hitota's theory. The modified combline bandpass filter discussed in this paper is built on the lumped model of a quarter-wave transmission line.

\section{Simulation and Fabrication}

To confirm the above theory, two bandpass filters working at $400 \mathrm{MHz}$ were designed and fabricated on a Taconic Teflon substrate with relative permittivity $\varepsilon_{r}=3.56$, thickness $=0.762 \mathrm{~mm}$ and the copper thickness $=34$ um. Firstly, a filter with the electrical length of the coupled line being $7^{\circ}$, named 7-degree filter, was designed. The value of the lumped capacitors was calculated to be $54.2 \mathrm{pF}$. The odd impedance of the coupled line $Z_{0 o}$ was $52.2 \Omega$ when the even mode impedance $Z_{0}$ was fixed at $70 \Omega$, making the coupling coefficient being 0.14. The length of the coupled line was calculated to be $9 \mathrm{~mm}$, while the width of each line was $1.1 \mathrm{~mm}$ and the slot of the coupled line was $0.7 \mathrm{~mm}$. Before fabrication, simulation using HFSS was done for a proper center frequency and a good insertion loss, which will be detailed in the following few graphs.

According to the simulation, the use of theoretical $54.2 \mathrm{pF}$ MIM capacitors has shifted center frequency to $350 \mathrm{MHz}$ due to the length of via hole. So here, $45 \mathrm{pF}$ capacitors were used to move it back to the predicted $400 \mathrm{MHz}$. As a rule of thumb, the midband of the proposed modified bandpass filter increases as the capacitor value decreases, and vice versa.

As mentioned in last section, the utilization of inter-stage transmission line between two neighboring stages is a new feature compared with the conventional combline bandpass filter. In this type of conventional filter, coupling between resonators is achieved by way of the fringing fields between resonator lines each other. However, the paralle short-ended coupled line with loading capacitors in the proposed filter is the equivalent of the inverter and the inverters were connected in original filter. Therefore, the neighboring line pair should be connected using inter-stage transmission line to limit the coupling within each pair of coupled line. If this inter-stage line is not included, shown in Fig. 13 (a), distortion appears. To show its importance more clearly, particular simulations in HFSS have been done. Fig. 13 (b), which gives the simulated results according to different distance $L_{i}$ between two stages, indicates that the performances are unsatisfactory. The distortion can be eliminated by using inter-stage transmission line, as illustrated in Fig. 14 (a). At first a 50 $\Omega$ transmission line with the length $L_{i}^{\prime}$ of $0.7 \mathrm{~mm}$ is added between two stages. However, distortion still occurs, as can be seen from the $S_{21}$ performance given in Fig. 14 (b) When the length is increased to $3 \mathrm{~mm}$, distortion gets less 
severe and $S_{21}$ improves. Here, a length of $5 \mathrm{~mm}$ connection transmission line is adopted in fabrication for a good insertion loss and proper center frequency.

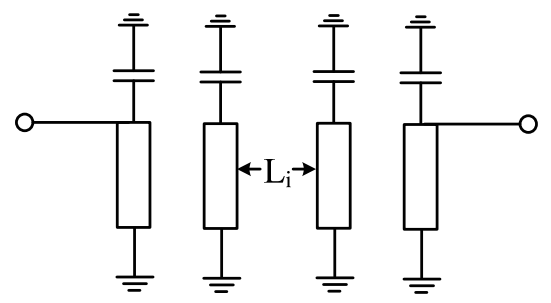

(a)

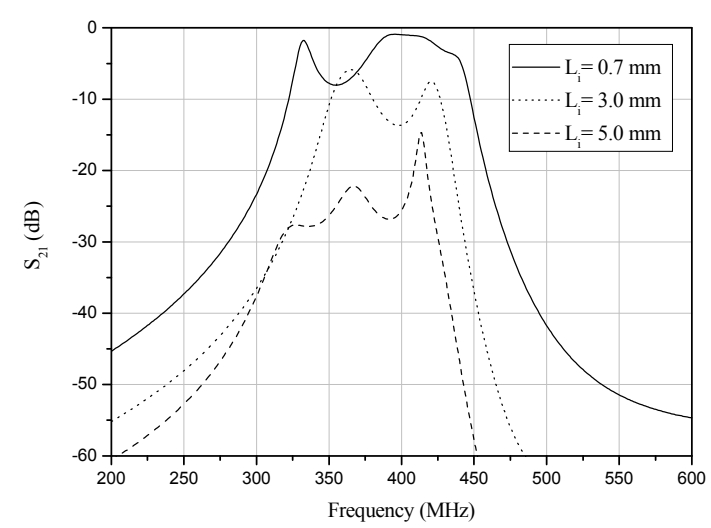

(b)

Fig. 13 Simulated results of 7-degree filter according to different inter-stage distance. (a) Circuit, (b) insertion loss.

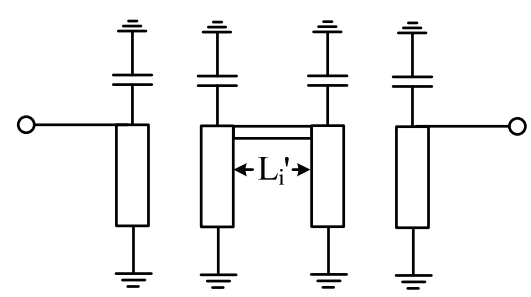

(a)

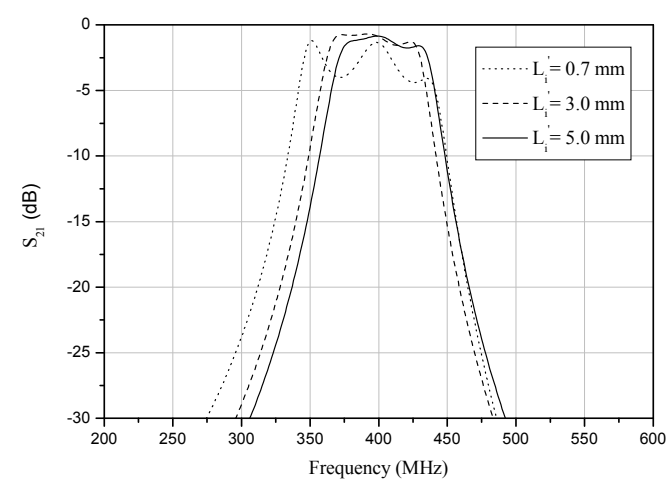

(b)

Fig. 14 The insertion loss performances of 7-degree filter according to different inter-stage connecting transmission line. (a) Circuit, (b) insertion loss.
It is insisted by some authors that the performances can be improved by tapping the input and output resonators( Kuo, 2003). However, in this proposed filter, connecting of different stages must be done at the fixed points for matching. As illustrated in Fig. 15 (a), connection points must be at $\mathrm{A}$ and $\mathrm{B}$. If $\mathrm{A}^{\prime}\left(\mathrm{A}^{\prime \prime}\right)$ and $\mathrm{B}$ ' (B") are used for connection, mismatching will occur between two neighboring stages. This point can be better supported by the simulation of the above 7-degree filter given in Fig. 15 (b), where $d_{1}=d_{2}=2 \mathrm{~mm}$.

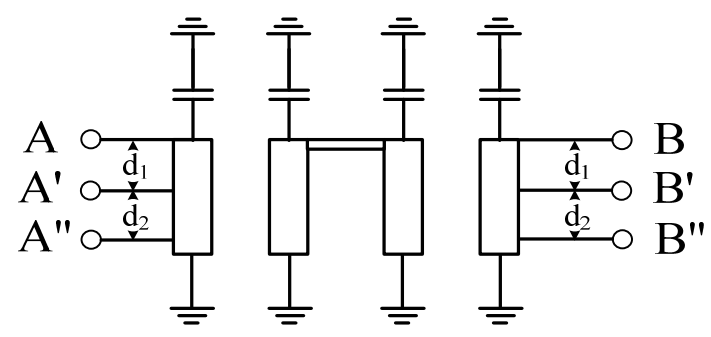

(a)

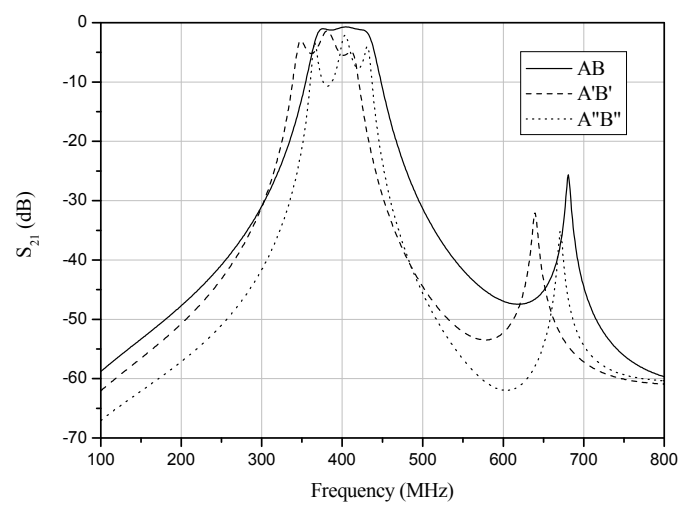

(b)

Fig. 15 (a) Position for connecting the input and output of independent bandpass filters, (b) simulated $S_{21}$ performances of 7-degree filter according to different connection points.

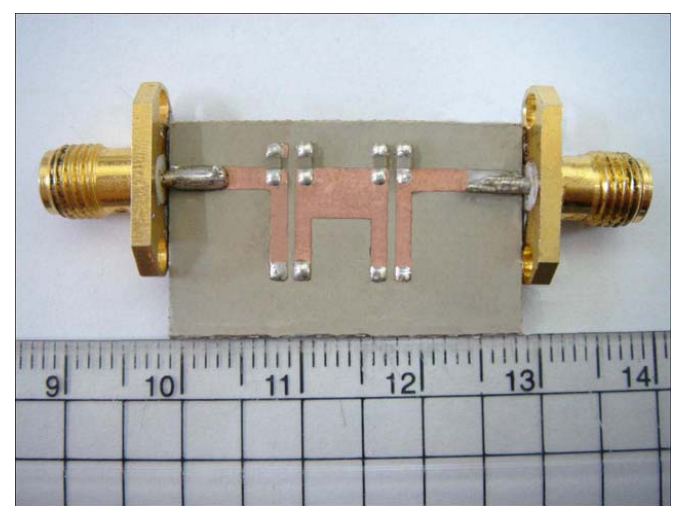

Fig. 16 The photo of the designed two-stage 7 -degree bandpass filter with a midband of $400 \mathrm{MHz}$. 
The size of the fabricated 7-degree filter is available in Fig. 16 , with one resonator taking up a circuit area of $9 \times 2.9 \mathrm{~mm}^{2}$. Fig. 17 gives the simulated results of the fabricated filter using Ansoft HFSS and the measured ones with Agilent 8722ES Network Analyzer. Some detailed performances are offered in Table 1. The measured center frequency is shifted from $400 \mathrm{MHz}$ to the left by $15 \mathrm{MHz}$ due to two reasons. The first one is the difference between MIM capacitors and lumped capacitors. In HFSS, MIIM capacitors should be used to carry out simulation. However, in fabrication only lumped capacitors were available. The MIM capacitor value calculated through $C=\varepsilon S / d$ is actually a little larger than that needed, due to the fringing effect. The other reason is that the lumped capacitors have an $\pm 5 \%$ error that can't be predicted. The spurious passband of the proposed passband filter does not appear or is very away from the passband, as a result of the small length of the coupled line, as shown in Fig. 17 (b). In fact, the first spurious was not observed even up to $11 \mathrm{GHz}$ during measurement.

Table 1 Measured performances of $400 \mathrm{MHz} 7$-degree filter

\begin{tabular}{|lr|}
\hline \multicolumn{2}{|c|}{$\begin{array}{c}\text { Characterstics of the } \\
\text { Fabricated Bandpass Filter }\end{array}$} \\
\hline Pass band (MHz) & 360 to 410 \\
Insertion Loss $(\mathrm{dB})$ & -3.1 at $\mathrm{f}_{0}$ \\
Attenuation $(\mathrm{dB})$ & -57 at $2 \mathrm{f}_{0}$ \\
& -55 at $3 \mathrm{f}_{0}$ \\
Characteristic impedance $(\mathrm{Ohm})$ & 50 \\
\hline
\end{tabular}

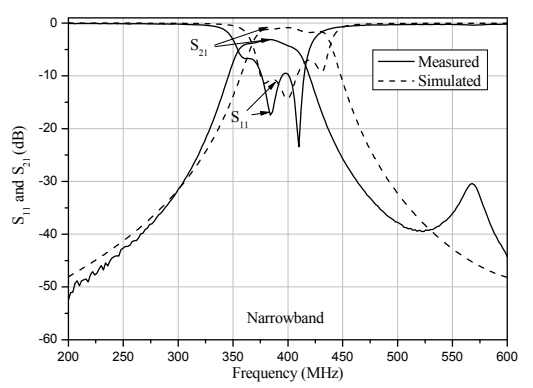

(a)

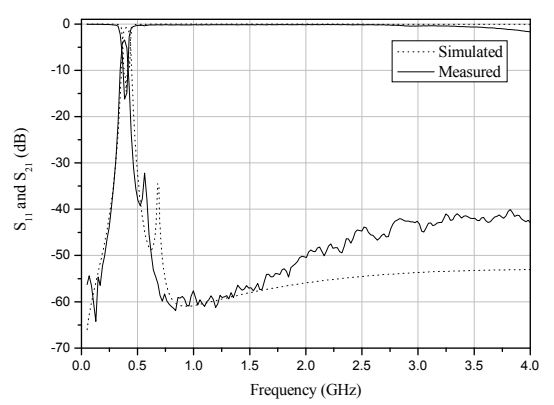

(b)

Fig. 17 Simulated and measured results of the designed two-stage 7-degree bandpass filter. (a) Narrowband response, (b) broadband response.
It is flexible to choose the electrical length of the coupled line. For a specified bandwidth, it is up to the designer. To prove that, another bandpass filter, with the electrical length of the coupled line being $5^{\mathrm{o}}$ has been designed. To get a similar bandwidth, the coupling coefficient $\mathrm{K}$ of the coupled line is made approximately to be 0.14 by choosing $Z_{0 e} 90 \Omega$ and $Z_{0 o} 68.5 \Omega$, respectively. The length of the coupled line is calculated to be $6.5 \mathrm{~mm}$, areduction of $28 \%$ with respect to the7-degree filter. Fig. 18 gives the simulated and measured results of the 5-degree filter, compared with the performances of 7-degree filter. The center frequency of the 5-degree filter is also shifted as a result of the inexact capacitor value.

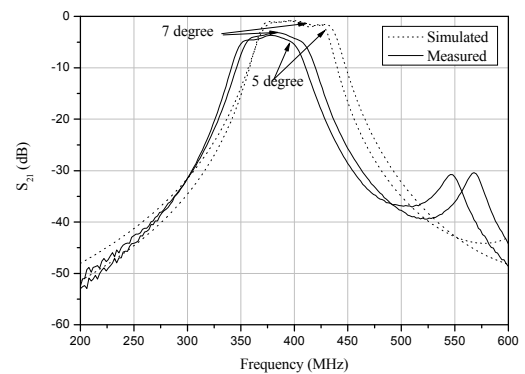

(a)

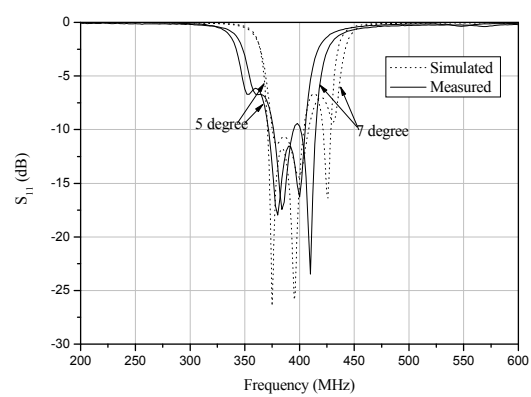

(b)

Fig. 18 Simulated and measured performances of $5^{-}$and 7-degree bandpass filter. (a) Insertion loss, (b) return loss.

On the other hand, the price paid for the realization of extreme miniaturization should be taken into account. When the size is miniaturized, the difference of $Z_{0 e}$ and $Z_{0 o}$ should be very small according to equation (11). In order to achieve it, the capacitance between the two strip conductors in odd mode should be as small as possible, resulting in wide slot of coupled line. This wide slot degrades the insertion loss performance because of weak coupling between the coupled line in spite of good matching. Therefore, a proper trade-off is indispensable in this case. Regarding the insertion loss of BPF, loss of a shorted stub operated as a distributed inductor is dominant in the 
Table 2 Comparison of sizes of different compact bandpass filters

\begin{tabular}{|c|c|c|c|c|c|c|c|c|c|}
\hline $\begin{array}{l}\text { Refer- } \\
\text { ence }\end{array}$ & $\begin{array}{c}\text { Band width } \\
\text { with- } 3 \mathrm{~dB} \\
(\mathrm{GHz})\end{array}$ & $\begin{array}{c}S_{11} \\
(\mathrm{~dB})\end{array}$ & $\begin{array}{c}S_{21} \\
(\mathrm{~dB})\end{array}$ & $\begin{array}{c}1^{\text {st }} \\
\text { Spurious } \\
\text { Passband } \\
(\mathrm{GHz})\end{array}$ & $\begin{array}{l}\text { Physical size of } \\
\text { a resonator } \\
(\mathrm{mm} \times \mathrm{mm})\end{array}$ & $\begin{array}{l}\text { Electrical } \\
\text { Length } \\
\text { (deg) }\end{array}$ & Structure & Technology & Year \\
\hline [4] & $2.11 \sim 2.17$ & $<-15$ & -1.7 & 5.2 & $10.8 \times 14.3$ & - & $2-\mathrm{D}$ & $\begin{array}{l}\text { Stepped-impedance } \\
\text { resonator -hairpin }\end{array}$ & 2006 \\
\hline [9] & $1.32 \sim 1.35$ & $<-20$ & -2.9 & 3.5 & $16.0 \times 6.5$ & 82.0 & $2-\mathrm{D}$ & Open-loop & 1997 \\
\hline [11] & $0.67 \sim 0.72$ & $<-24$ & -0.6 & 1.3 & $30.6 \times 8.0$ & 32.4 & $2-\mathrm{D}$ & Slow-wave & 2006 \\
\hline [16] & $2.30 \sim 2.65$ & $<-20$ & -1.7 & - & $3.2 \times 2.5 \times 1.2 *$ & 22.8 & LTCC & Combline & 2003 \\
\hline [17] & $2.4 \sim 2.5$ & $<-20$ & -1.4 & - & $2.8 \times 2.3 \times 0.7 *$ & $12 * *$ & LTCC & Combline & 2006 \\
\hline [25] & $0.66 \sim 0.74$ & $<-10$ & -1.2 & 2.8 & $55.0 \times 35.0$ & 90.0 & $2-\mathrm{D}$ & Inter-digital & 2004 \\
\hline [26] & $2.25 \sim 2.33$ & $<-24$ & -5.8 & - & $10.2 \times 10.0$ & 90.0 & $2-\mathrm{D}$ & $\begin{array}{c}\text { Stepped-impedance } \\
\text { resonator }\end{array}$ & 2006 \\
\hline [27] & $1.90 \sim 2.10$ & $<-10$ & -1.9 & 6.0 & $18.0 \times 7.4$ & 72.2 & $2-\mathrm{D}$ & Parallel-coupled & 2005 \\
\hline [28] & $2.28 \sim 2.52$ & $<-12$ & -2.9 & 6.0 & $5.0 \times 5.0$ & - & $2-\mathrm{D}$ & $\begin{array}{l}\text { Metamaterial } \\
\text { resonator }\end{array}$ & 2006 \\
\hline \multirow{2}{*}{$\begin{array}{l}\text { This } \\
\text { work }\end{array}$} & $0.36 \sim 0.41$ & $<-9$ & -3.1 & Not found & $9.0 \times 2.9$ & 7.0 & $2-\mathrm{D}$ & \multirow{2}{*}{ Modified combline } & 2010 \\
\hline & $0.35 \sim 0.40$ & $<-10$ & -3.9 & Not found & $6.5 \times 2.3$ & 5.0 & $2-\mathrm{D}$ & & 2010 \\
\hline
\end{tabular}

*: 3-dimensional circuit size using low temperature co-fired (LTCC) technology

$$
\text { **: } 2 \text { poles are used }
$$

entire loss and expressed as follows: (Kang, 2008)

$$
S_{21}=\frac{1}{1+\frac{Z_{o e}}{2}\left(\frac{\alpha l}{Z_{o e} \sin ^{2} \beta l}\right)}
$$

where $\alpha$ is the attenuation constant of shunt stub, $\beta$ is the propagation constant, and $l$ is the length of stub.

According to (16), insertion loss was inclined to be depending on the extent of the miniaturization of stub length. Insertion loss with 5-degree electrical length became worse about $-0.8 \mathrm{~dB}$ than one with 7 -degree. Therefore, for the highly miniaturized filter design, the sacrifice of insertion loss will be inherently unavoidable.

Finally, a size comparison of the different types of compact filters is made here to show the advantage of the proposed compact bandpass filter, as illustrated in Table 2.

\section{Conclusion}

In this paper, a novel combline filter is successfully developed using 2-dimensional planar coupled line structure. Compared with the traditional combline filter, the proposed filter takes up a much smaller circuit area. This is because the electrical length of the coupled line, which determines the size of combline filter, can be reduced to just a few degrees, with the help of lumped capacitors. Suppression of the spurious passband is another advantage of the modified combline filter. Measured results of the fabricated $400 \mathrm{MHz}$ filter matched very well with the simulated performances, which verified the validity of the size-reduction method. This size-reduction concept can also be extended to the MMIC field.

\section{Reference}

[1] Cohn S. B.(1958), "Parallel coupled transmission line resonators," IRE MTT Vol. 6-4, pp. 223-231

[2] Djaiz A. and Denidni T. A. (2006), "A new compact microstrip two-layer bandpass filter using apertured-coupled SIR-hairpin resonators with transmission zeros," IEEE Trans. Microwave Theory Tech., vol.54, no.5, pp.1929-1936

[3] Garcia J. G. and Bonache J. (2006), "Miniaturized microstrip and CPW filters using coupled metamaterial resonators," IEEE Trans. Microwave Theory Tech., vol. 54. no. 6. pp. 2628-2635

[4] Hong J. S. and Lancaster M.J. (1998), "Cross-coupled microstrip hairpin-resonator filters," IEEE Trans. Microwave Theory Tech., vol. 46, no. 1, pp. 118-122.

[5] Hong J. S. and Lancaster M. J. (1997), "Theory and experiment of novel microstrip slow-wave open-loop resonator filters," IEEE Trans. Microwave Theory Tech., vol. 45, no. 12, pp. $2358-2365$ 
[6] Hong J. S. and Lancaster M. J. (1996), "Cross-coupled microstrip square open-loop resonators for cross-coupled planar microwave filters," IEEE Trans. Microwave Theory Tech., vol. 44, no. 11, pp. 2099 - 2109

[7] Ju L. H., Bhattacharya P., Katehi L.P.B. (1984), " $\mathrm{X}$-band and $\mathrm{K}$-band lumped Wilkinson power dividers with a micromachined technology" IEEE MTT-S Digest, pp.409-411

[8] Kang I. H. and Park J. S. (2003), “A reduced-size power divider using the coupled line equivalent to a lumped inductor," Microwave Journal, vol. 46, no. 7

[9] Kang I. H. and Xu H. Y. (2007), "An Extremely Miniaturized Microstrip Bandpass Filter," Microwave Journal, vol. 50, no. 5, pp. 228 - 233

[10] Kang, I., Wang, S., Yun, Y., Jhang, H. (2008) "Theoretical analysis on attenuation of the $5 \mathrm{GHz}$ miniaturized GaAs MMIC bandpas filter", Microwave Journal, August,

[11] Kuo J. T., Chen S. P. and Jiang M. (2003), "Parallel-coupled microstrip filters with over-coupled end stages for suppression of spurious responses," IEEE Trans. Microwave Theory Tech., vol. 13, no. 10, pp. $440-442$

[12] Kuo J. T., Jiang M. and Chang H. J. (2004), "Design of parallel-coupled microstrip filters with suppression of spurious resonances using substrate suspension," IEEE Trans. Microwave Theory Tech., vol .52, no. 1, pp.83-89

[13] Kuo J. T. and Shih E. (2003), "Microstrip stepped impedance resonator bandpass filter with an extended optimal rejection bandwidth," IEEE Trans. Microwave Theory Tech., vol.51, no.5, pp. 1554-1559

[14] Kong Y. W. and Chew S.T. (2004), "A miniaturizedend-coupled filter using stepped-impedance resonators," Micro. Opt. Technol. .Lett., Vol. 46, no. 2, pp. 97-99

[15] Lin Y. S. and Wang C. H. (2005), "Novel compactparallel-coupled microstrip bandpass filters with lumped-element $\mathrm{K}$-inverters," IEEE Trans. Microwave Theory Tech., vol. 53, no. 7, pp. $2324-2328$

[16] Lopetegi T. and Laso M. A. G. (2001), “New microstrip wiggly line filters with spurious passband suppression," IEEE Trans. Microwave Theory Tech., vol. 49, no. 9, pp. 1593-1598

[17] Matthaei G., Young L. and Jones E.M.T. (1980), Microwave Filters, Impedance Matching Networks and Coupling Structures, Artech House Inc., Norwood, MA 1980, p. 500.

[18] Matthaei G., Young L. and Jones E.M.T. (1980), Microwave Filters, Impedance Matching Networks and
Coupling Structures, Artech House Inc., Norwood, MA 1980, p. 433

[19] Makimoto M. and Yamashita S. (1980), "Bandpass filters using parallel coupled strip-line stepped impedance resonators," IEEE Trans. Microwave Theory Tech., vol. MTT-28, no. 12, pp. 1413 - 1417

[20] Pang H. K. and Ho K. M. (2004), “A compact microstrip $N / 4$-SIR interdigial bandpass filter with extended stopband," IEEE MTT-S Digest, pp. 1621 - 1624

[21] Pistono E., Robert M. and Duvillaret L. (2006), "Compact fixed and tune-all bandpass filters based on coupled slow-wave resonators," IEEE Trans. Microwave Theory Tech., vol. 54, no. 6, pp. 2790-2799

[22] Sagawa M., Takahashi K., and Makimoto M. (1989), "Miniaturized hairpin resonator filers and their application to receiver fron-end MIC's," IEEE Trans. Microwave Theory Tech., vol..37, no.12, pp. 1991-1997

[23] Tang C. W., Lin Y. C. and Chang C. Y. (2003), "Realization of transmission zeros in combline filters using an auxiliary inductively coupled ground plane," IEEE Trans. Microwave Theory Tech., vol.51, no.10, pp.2112-2118

[24] Tang C. W and S. F, You(2006), "Design Methodologies of LTCC bandpass filters, diplexer, and triplexer with transmission zeros," IEEE Trans. Microwave Theory Tech., vol. 54, no. 2, pp. 717-723

[25] Wang S. M., Chi C. H. and Hsieh M. Y. (2005), "Miniaturized spurious passband suppression microstrip filter using meandered parallel coupled lines," IEEE Trans. Microwave Theory Tech, vol. 53, no. 2, pp. $747-753$

[26] Wang X., Liu P., and Li Y. (2004), "New compact configuration of a stepped-impedance ceramic bandpass filter," Micro. Opt. Technol.Lett., Vol. 41, no. 2, pp. $146-149$

[27] Wang, X. G., Yun, Y. and Kang, I. H.(2009), "Compact Multi-harmonic Suppression LTCC Bandpass Filter Using Parallel Short-ended Coupled Line Structure," ETRI Journal, vol. 30, no.3, June p. 254-262.

[28] Yao H. W. and Wang C., and Zaki K. A. (1996), c"Quarter wavelength ceramic combline filters," IEEE Trans. Microwave Theory Tech., vol. 44, no. 12, pp. 2673-2679

[29] Zhang H. L. and Chen K. J. (2006), "Miniaturized coplanar waveguide bandpass filters using multisection stepped-impedance resonators," IEEE Trans. Microwave Theory Tech., vol. 54, no. 3, pp. 1090-1095

Received 31 March 2010

Revised 27 July 2010

Accepted 30 August 2010 